\title{
Biased Metropolis-Heatbath Algorithms for Lattice Gauge Theory
}

\author{
Alexei Bazavov* \\ Department of Physics, Florida State University, Tallahassee, FL 32306-4350 \\ School of Computational Science, Florida State University, Tallahassee, FL 32306-4120 \\ E-mail: bazavovecsit.fsu.edu
}

\section{Bernd A. Berg}

Department of Physics, Florida State University, Tallahassee, FL 32306-4350

School of Computational Science, Florida State University, Tallahassee, FL 32306-4120

E-mail: berg@csit.fsu.edu

\begin{abstract}
It is illustrated for 4D $S U(2)$ lattice gauge theory that sampling with a biased Metropolis scheme is essentially equivalent to using the heatbath algorithm. Only, the biased Metropolis method can also be applied when an efficient heatbath algorithm does not exist. Other cases for which the use of the biased Metropolis-heatbath algorithm is beneficial are briefly summarized.
\end{abstract}

XXIIIrd International Symposium on Lattice Field Theory

25-30 July 2005

Trinity College, Dublin, Ireland

${ }^{*}$ Speaker. 


\section{Introduction}

In this talk we introduce a Metropolis-type updating procedure, which uses approximate heatbath probabilities for its proposals, so that an acceptance rate close to 1 is achieved without any undue shrinking of the proposal range. This introduces a bias in the probability ratio of the Metropolis accept/reject step, and we call the algorithm Biased Metropolis-Heatbath Algorithm (BMHA), while we use the notation HBA for HeatBath Algorithm. Details of the application of the BMHA to $S U(2)$ and $U(1)$ gauge theories are already published in [1].

\section{2. $S U(2)$ Pure Gauge Theory Example}

Here we explain the $S U(2)$ example. The action is

$$
S(\{U\})=\frac{1}{N_{c}} \sum_{\square} \operatorname{Re} \operatorname{Tr}\left(U_{\square}\right)
$$

with $U_{\square}=U_{i_{1} j_{1}} U_{j_{1} i_{2}} U_{i_{2} j_{2}} U_{j_{2} i_{1}}$, where the sum is over all plaquettes of a $4 \mathrm{D}$ simple hypercubic lattice, $i_{1}, j_{1}, i_{2}$ and $j_{2}$ label the sites circulating about the plaquette and $U_{j i}$ is a $S U(2)$ matrix $\left(N_{c}=2\right)$ associated with the link $\langle i j\rangle$. The reversed link is associated with the inverse matrix. While working at a particular link $\langle i j\rangle$, we need only to consider the contribution to $S$, which comes from the staples containing this link. We denote by $U_{\sqcup, k}, k=1, \ldots, 6$, the products which interact with the link in question. Then the probability density of this link matrix is

$$
d P(U) \sim d U \exp \left[\frac{\beta_{g}}{N_{c}} \operatorname{Re} \operatorname{Tr}\left(U \sum_{k=1}^{6} U_{\sqcup, k}\right)\right] .
$$

Using the property of $S U(2)$ group elements that any sum of them is proportional to another $S U(2)$ element we defi ne a $S U(2)$ matrix $U$ by

$$
U_{\sqcup}=\frac{1}{s_{\sqcup}} \sum_{k=1}^{6} U_{\sqcup, k}, \quad s_{\sqcup}=\sqrt{\operatorname{det}\left(\sum_{k=1}^{6} U_{\sqcup, k}\right)} .
$$

Let $V=U U_{\sqcup}$, using the invariance of the group measure, $d V=d U$, one fi nds

$$
d P(V) \sim d V \exp \left[\frac{\beta_{g}}{2} s_{\sqcup} \operatorname{Re} \operatorname{Tr}(V)\right] .
$$

In the basis of Pauli matrices, $\vec{\sigma}, V$ is conveniently parametrized:

$$
V=a_{0} I+i \vec{a} \cdot \vec{\sigma}, a_{0}^{2}+\vec{a}^{2}=1 \Rightarrow \operatorname{Tr}(V)=2 a_{0} .
$$

The group measure is $d V=\sqrt{1-a_{0}^{2}} d a_{0} d \Omega$ with $d \Omega$ being solid angle of the three dimensional $\vec{a}$. Let us denote $\alpha=\beta_{g} s_{\sqcup}$, then

$$
d P(V) \sim d \Omega d a_{0} \sqrt{1-a_{0}^{2}} \exp \left(\alpha a_{0}\right) .
$$




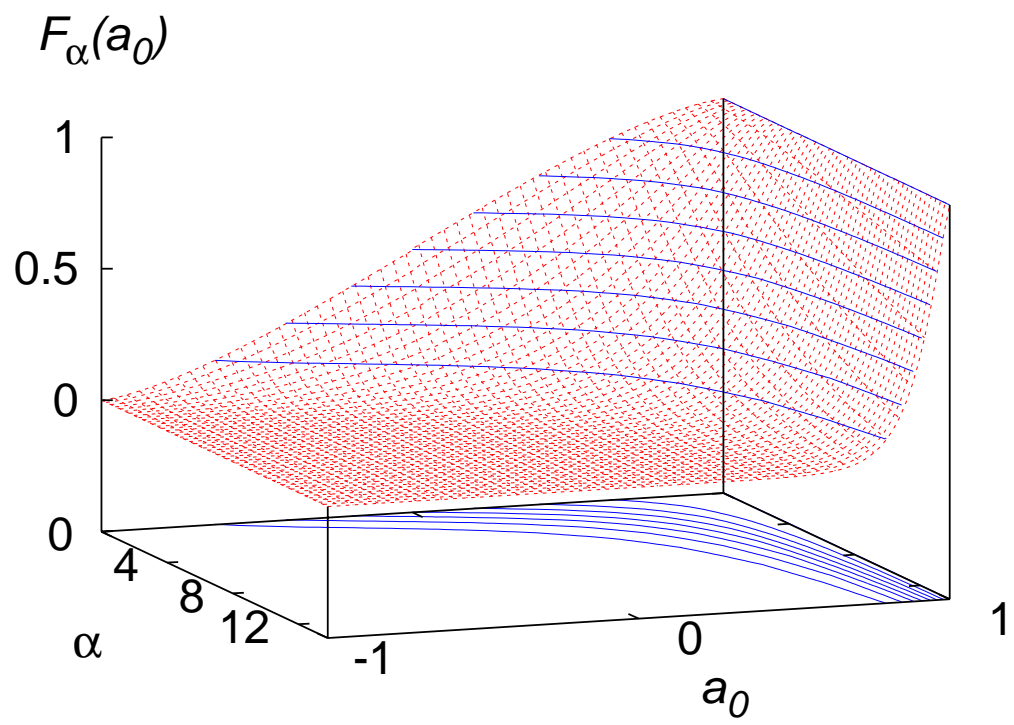

Figure 1: Cumulative distribution function $F_{\alpha}\left(a_{0}\right)$ with the level map in $\alpha-a_{0}(x-y)$ plane.

As nothing depends on the solid angle, $\vec{a}$ is generated uniformly on a sphere of a radius $\sqrt{1-a_{0}^{2}}$ and the main step is to generate $a_{0}$ in the interval $-1 \leq a_{0} \leq 1$ with probability density

$$
P_{\alpha}\left(a_{0}\right) \sim \sqrt{1-a_{0}^{2}} \exp \left(\alpha a_{0}\right) .
$$

The cumulative distribution function $(\mathrm{CDF})$ is

$$
F_{\alpha}\left(a_{0}\right)=N_{\alpha} \int_{-1}^{a_{0}} d a_{0}^{\prime} \sqrt{1-a_{0}^{\prime 2}} \exp \left(\alpha a_{0}^{\prime}\right)
$$

where $N_{\alpha}$ ensures the normalization $F_{\alpha}(1)=1$ and, evidently, $P_{\alpha}\left(a_{0}\right)=d F_{\alpha}\left(a_{0}\right) / d a_{0}$.

$F_{\alpha}\left(a_{0}\right)$ is a function of two variables: the parameter $\alpha$, which incorporates the effect of interaction with the neighbors, and $a_{0}$, the variable being updated. In the following we will associate $\alpha$ to the $x$-, $a_{0}$ to the $y$-, and $F_{\alpha}\left(a_{0}\right)$ to the $z$-axis. Also, we will consider $S U(2)$ gauge theory at a coupling $\beta_{g}=2.3$, close to the critical point. In this case $0 \leq \alpha \leq 6 \beta_{g}=13.8$. With these conventions $F_{\alpha}\left(a_{0}\right)$ is plotted on Fig. 1. Contour lines on the surface represent levels where $F_{\alpha}\left(a_{0}\right)$ increases from 0 to 1 by a chosen constant value (in this case 1/8). Lines in $\alpha-a_{0}(x-y)$ plane are projections of these contours and constitute a level map similar to those used to encode height on maps in geography. What we need to construct a BMHA is a discretized version of this level map.

\section{Constructing the Algorithm}

\subsection{Heatbath Algorithm}

The HBA generates $a_{0}$ for a given $\alpha$ by converting a uniformly distributed random number $0 \leq z<1$ into $a_{0}=F_{\alpha}^{-1}(z)$. For $S U(2)$ it was fi rst introduced by Creutz [3] and improved in Ref. [3] and [4]. As $F_{\alpha}^{-1}(z)$ is approximated one needs a repeat until accepted (RUA) step to generate the correct distribution. The acceptance rate $(A R)$ of the updates, defi ned as the ratio of updated links over the number of visited links, is always 1 for the HBA [5]. 


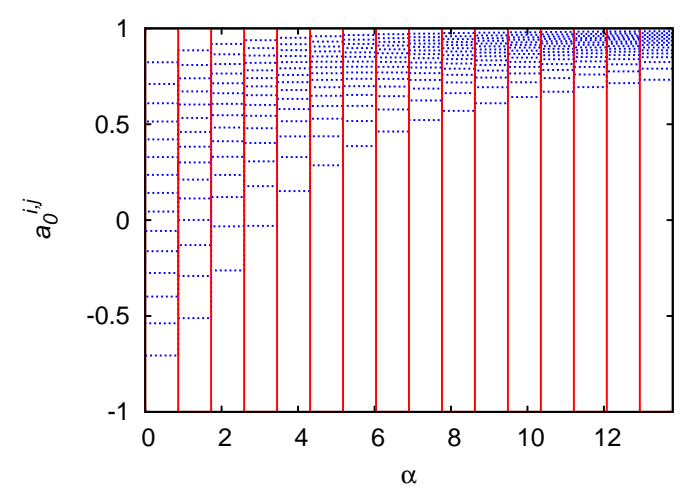

Figure 2: $m \times n$ partitioning of $\triangle a_{0}^{i, j}$ for $S U(2)$ at $\beta_{g}=2.3$ for the table values discussed in the text.

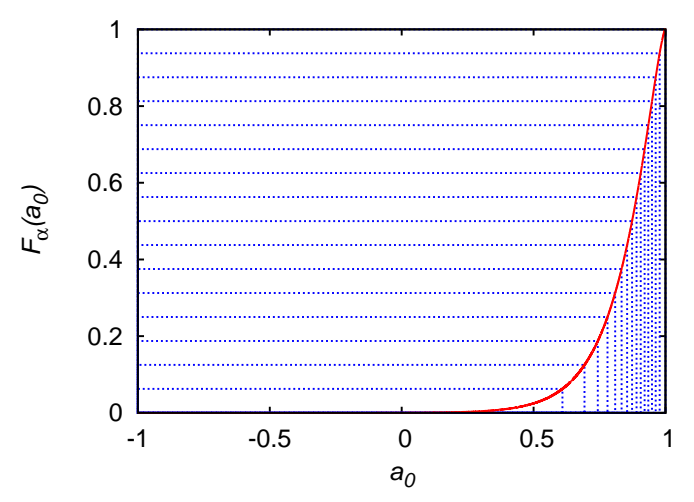

Figure 3: Discretization of the cumulative distribution function $F_{\alpha^{11}}\left(a_{0}\right)$ for $S U(2)$ at $\beta_{g}=2.3$.

\subsection{Metropolis Algorithm}

In the conventional Metropolis scheme $a_{0, \text { new }}$ is generated uniformly in the range $[-1,1]$ and then accepted with probability

$$
p_{\text {Met }}=\min \left\{1, \frac{P_{\alpha}\left(a_{0, \text { new }}\right)}{P_{\alpha}\left(a_{0, \text { old }}\right)}\right\}=\min \left\{1, \frac{\sqrt{1-a_{0, \text { new }}^{2}} \exp \left(\alpha a_{0, \text { new }}\right)}{\sqrt{1-a_{0, \text { old }}^{2}} \exp \left(\alpha a_{0, \text { old }}\right)}\right\} .
$$

It may have a low AR in the region of interest. Possible cures are to decrease the proposal range, which makes the moves small, or multi-hit Metropolis, which needs a fi xed number of hits (i.e. cannot be transformed into a RUA step).

\subsection{Biased Metropolis-Heatbath Algorithm}

Let us discretize the parameter $\alpha\left(x\right.$ axis) into $m=2^{n_{1}}=16\left(n_{1}=4\right)$ bins. For simplicity we choose equidistant partitioning, although other discretizations are possible too. In each $\alpha^{i}$ bin we discretize $F_{\alpha^{i}}\left(a_{0}\right)$ ( $z$ axis) into $n=2^{n_{2}}=16\left(n_{2}=4\right)$ values and store $a_{0}^{i, j}$ which satisfy the condition: $j / n=F_{\alpha^{i}}\left(a_{0}^{i, j}\right)$. In this way we achieve a discretized version of the level map at the bottom of Fig. 1, which is shown in Fig. 2.

Two two-dimensional arrays are needed: one for storing $a_{0}^{i, j}$ (levels themselves) and another for $\triangle a_{0}^{i, j}=a_{0}^{i, j}-a_{0}^{i, j-1}$ (distances between levels). Let us assume that for a link being updated $\alpha$ falls into the 11th bin, so $i=11$. Finding $i$ is achieved by an operation of the form: integer $\left[m \alpha / \alpha_{\max }\right]$ with $\alpha_{\max }=6 \beta_{g}=13.8$ for $\beta_{g}=2.3$. This is our fi rst step.

Then $F_{\alpha^{11}}\left(a_{0}\right)$ is given by a cross section in the $y-z$ plane shown on Fig. 3. The next step is to determine the bin label $j_{\text {old }}$ which belongs to the (known) value $a_{0, \text { old }}$. This can be done in $n_{2}$ steps using the recursion $j \rightarrow j+2^{i_{2}} \operatorname{sign}\left(a_{0}-a_{0}^{i, j}\right), i_{2} \rightarrow i_{2}-1$. Once $j_{\text {old }}$ is known it gives a length of the bin: $\triangle a_{0}^{i, j_{o l d}}$.

The next step is to propose $a_{0, \text { new }}$. Two uniform random numbers $\left(r_{1}\right.$ and $\left.r_{2}\right)$ are needed: one to pick up an integer $j_{\text {new }}$ in the range $1, \ldots, n$ (as $j_{\text {new }}=$ integer $\left[n r_{1}\right]$ ), another to propose 
Table 1: Efficiency of the $S U(2)$ algorithms on a $4 \times 16^{3}$ lattice at $\beta_{g}=2.3$.

\begin{tabular}{|c|c|c|c|}
\hline & HBA [3, $₫]$ & Metropolis & BMHA \\
\hline CPU time & $194,873[\mathrm{~s}]$ & $181,321[\mathrm{~s}]$ & $199,244[\mathrm{~s}]$ \\
\hline Acceptance rate & $1(1.043$ proposals $)$ & 0.111 & 0.975 \\
\hline$\left\langle\operatorname{Tr}\left(U_{\square}\right) / 2\right\rangle$ & $0.603147(17)$ & $0.603066(52)$ & $0.603111(21)$ \\
\hline$\tau_{\text {int }}$ & $49.8(3.5)$ & $409(66)$ & $48.2(3.8)$ \\
\hline
\end{tabular}

$a_{0, \text { new }}=a_{0}^{i, j_{\text {new }}-1}+r_{2} \triangle a_{0}^{i, j_{\text {new }}}$. Then the new value of a variable is accepted with probability:

$$
p_{B M A}=\min \left\{1, \frac{P_{\alpha}\left(a_{0, \text { new }}\right)}{P_{\alpha}\left(a_{0, \text { old }}\right)} \cdot \frac{\triangle a_{0}^{i, j_{\text {new }}}}{\triangle a_{0}^{i, j_{\text {old }}}}\right\}=\min \left\{1, \frac{\sqrt{1-a_{0, \text { new }}^{2}} \exp \left(\alpha a_{0, \text { new }}\right)}{\sqrt{1-a_{0, \text { old }}^{2}} \exp \left(\alpha a_{0, \text { old }}\right)} \cdot \frac{\triangle a_{0}^{i, j_{\text {new }}}}{\triangle a_{0}^{i, j_{\text {old }}}}\right\} .
$$

$p_{B M A}$ in (3.2) differs from $p_{M e t}$ in (3.1) by a bias $\triangle a_{0}^{i, j_{\text {new }}} / \triangle a_{0}^{i, j_{\text {old }}}$.

We need to distinguish two issues here. First, the underlined updating procedure stays fully equivalent to the conventional Metropolis algorithm for any discretization $a_{0}^{i, j}$. Detailed balance for (3.2) can be proven the same way as for usual Metropolis without explicit knowledge of $\triangle a_{0}^{i, j}$. Choosing, for example, equidistant partitioning for $a_{0}\left(\triangle a_{0}^{i, j}=\triangle a_{0}^{i, k}\right.$ for any $\left.j, k\right)$ would turn the bias into 1 and simply get us back to original Metropolis algorithm. Hence, the bias influences only the acceptance rate in (3.2). This draws us to the second issue: when partitioning $a_{0}^{i, j}$ is achieved by discretizing the cumulative distribution function, then in the limit $m, n \rightarrow \infty$ the BMHA approaches the HBA and the acceptance rate approaches 1 . The limit $m \rightarrow \infty$ simply means $\alpha^{i} \rightarrow \alpha$, the actual value. For the $n \rightarrow \infty$ limit let us turn to the Fig. 3. The discretization step on $z=F_{\alpha}\left(a_{0}\right)$ axis is $\triangle z=1 / n$ and $a_{0}=F_{\alpha}^{-1}(z)$. Therefore, as $n \rightarrow \infty, \triangle z \rightarrow 0$ :

$$
\frac{\triangle a_{0}}{\triangle z} \rightarrow \frac{d}{d z} F_{\alpha}^{-1}(z)=\left(\frac{d}{d a_{0}} F_{\alpha}\left(a_{0}\right)\right)^{-1}=\frac{1}{P_{\alpha}\left(a_{0}\right)} .
$$

Then,

$$
\frac{P_{\alpha}\left(a_{0, \text { new }}\right)}{P_{\alpha}\left(a_{0, \text { old }}\right)} \frac{\triangle a_{0}^{i, j_{\text {new }}}}{\triangle a_{0}^{i, j_{\text {old }}}}=\frac{P_{\alpha}\left(a_{0, \text { new }}\right)}{P_{\alpha}\left(a_{0, \text { old }}\right)} \frac{\triangle a_{0}^{i, j_{\text {new }}} / \triangle z}{\triangle a_{0}^{i, j_{\text {old }}} / \triangle z} \rightarrow \frac{P_{\alpha}\left(a_{0, \text { new }}\right)}{P_{\alpha}\left(a_{0, \text { old }}\right)} \frac{1 / P_{\alpha}\left(a_{0, \text { new }}\right)}{1 / P_{\alpha}\left(a_{0, \text { old }}\right)}=1 .
$$

The table building process and the updating procedure are discussed in a more formal way in [1]].

\section{Performance}

In our simulations we used a fi ner discretization, $m=32$ and $n=128$. Table 1 illustrates the performance of the $S U(2)$ BMHA for a long run on a $4 \times 16^{3}$ lattice at $\beta_{g}=2.3$. At this coupling the system exhibits critical slowing down, because of its neighborhood to the deconfi ning phase transition (see for instance [6] and references therein). We used 16,384 sweeps for reaching equilibrium and, subsequently, $32 \times 20,480$ sweeps for measurements. Simulations were performed on $2 \mathrm{GHz}$ Athlon PCs with the -O2 option of the (freely available) g77 Fortran compiler.

Our comparison is with the Fabricius-Haan-Kennedy-Pendleton HBA [3, 那, which at this coupling is more effi cient than Creutz's HBA [4], and with the conventional Metropolis. A direct 
measure for the performance of an algorithm is the integrated autocorrelation time $\tau_{\text {int }}$. It is given in the Table 1 for the Wilson plaquette together with the expectation value of this operator. Error bars are given in parenthesis and apply to the last digits. They are calculated with respect to 32 bins (jackknife bins in case of $\tau_{\text {int }}$ ), relying on the data analysis software of 77 .

\section{Summary and Conclusions}

Using BMHA may be benefi cial in several lattice gauge theory situations:

- When a HBA implementation exists, but the inversion of the cumulative distribution function in question is relatively ineffi cient. An example is $U(1)$ lattice gauge theory explored in [1].

- When a HBA implementation does not exist. An example is $S U(2)$ lattice gauge theory in the mixed fundamental-adjoint representation (work in progress [9]).

- In multi-variable case to which the BMHA generalizes for the price of increasing dimensions of the tables.

- In (checkerboard) parallelization the updating speed of the BMHA is uniform over all nodes. This is not the case for a HBA if a RUA step is involved (as for $S U(2)$ ).

When the canonical CDF similar to (2.8) is a priori unknown a HBA becomes impossible, but there are still good chances for a Biased Metropolis Algorithm (BMA). In this case an estimator of the CDF may be constructed empirically as it was explored for biophysical applications in [8], where a BMA of the type discussed here was fi rst proposed within a Rugged Monte Carlo scheme.

\section{Acknowledgments}

The authors would like to thank Wolfhard Janke and his group at Leipzig University for their kind hospitality during the summer 2005. This work was in part supported by the US Department of Energy under contract DE-FG02-97ER41022.

\section{References}

[1] A. Bazavov and B.A. Berg, Phys. Rev. D 71, 114506 (2005)

[2] M. Creutz, Phys. Rev. D 21, 2308 (1980).

[3] K. Fabricius and O. Haan, Phys. Lett. B 143, 459 (1984).

[4] A.D. Kennedy and B.J. Pendleton, Phys. Lett. B 156, 393 (1985).

[5] In some of the literature the quantity $1 /$ (average number of RUA heat bath iterations per update) is also called acceptance rate. It should not be confused with the acceptance rate defined here.

[6] J. Fingberg, U. Heller, and F. Karsch, Nucl. Phys. B 392, 493 (1993).

[7] B.A. Berg, Markov Chain Monte Carlo Simulations and Their Statistical Analysis, World Scientific, 2004.

[8] B.A. Berg, Phys. Rev. Lett 90, 180601 (2003); B.A. Berg and H.-X. Zhou, Phys. Rev. E 72, 016712 (2005).

[9] A. Bazavov, B.A. Berg and U. Heller, in preparation. 